\title{
Коромыслов К.Е.
}

\section{Исследование способов обнаружения метода сетевой стеганографии, основанного на преднамеренных временных задержках}

Санкт-Петербургский государственный университет телекоммуникаций им. проф. М. А. Бонч-Бруевича (Россия, Санкт-Петербург)

doi: $10.18411 / \mathrm{j}-02-2021-09$

idsp: ljournal-02-2021-09

\section{Аннотация}

В данном докладе, будут представлены основные сведения о методе сетевой стеганографии, основанном на преднамеренных временных задержках. Основной акцент будет сделан на описание данного метода, его особенности, практическую реализацию и способы обнаружения. В результате описаны два основных способа обнаружения данного метода, а также экспериментальная реализация одного из них.

Ключевые слова: методы сетевой стеганографии, изменение структуры передачи пакетов, Wireshark, Pcap4j.

\section{Abstract}

This report will provide basic information on the network steganography method based on deliberate time delays. The main attention will be paid to the description of this method, its features, practical implementation and detection methods. As a result, two main methods of detecting this method are described, as well as the experimental implementation of one of them.

Keywords: methods of network steganography, changing the packet transfer structure, Wireshark, Pcap4j.

Основная особенность сетевой стеганографии заключается в том, что скрытая информация передается по компьютерным сетям с учетом особенностей работы протоколов передачи данных. Чаще всего принцип работы методов сетевой стеганографии обусловлен изменением параметров передачи одного или нескольких сетевых протоколов модели OSI или TCP/IP. Большинство данных подходов создает значительные излишние подозрения, поскольку напрямую изменяются поля заголовков пакетов. Метод, основанный на преднамеренных временных задержках, отличается высоким уровнем скрытности, поскольку временные задержки считаются повседневным явлением в поведении сетей и не вызывают лишних подозрений. $[1,2,3$, 4]

Описание метода, основанного на преднамеренных временных задержках.

Данный метод основывается на модуляции времени ожидания между пакетными передачами. Он фактически использует обычный трафик, внося задержки по времени между передаваемыми пакетами. Например, для реализации азбуки Морзе, можно предположить, что короткая временная задержка между двумя последовательными пакетами кодирует двоичный ноль, а длительная временная задержка кодирует двоичную единицу.

В общем случае, предположим, что Алиса собирается обменяться информацией с Бобом и отправляет данные со своего компьютера путем кодификации информации в виде временных задержек между пакетами, (рисунок 1). 


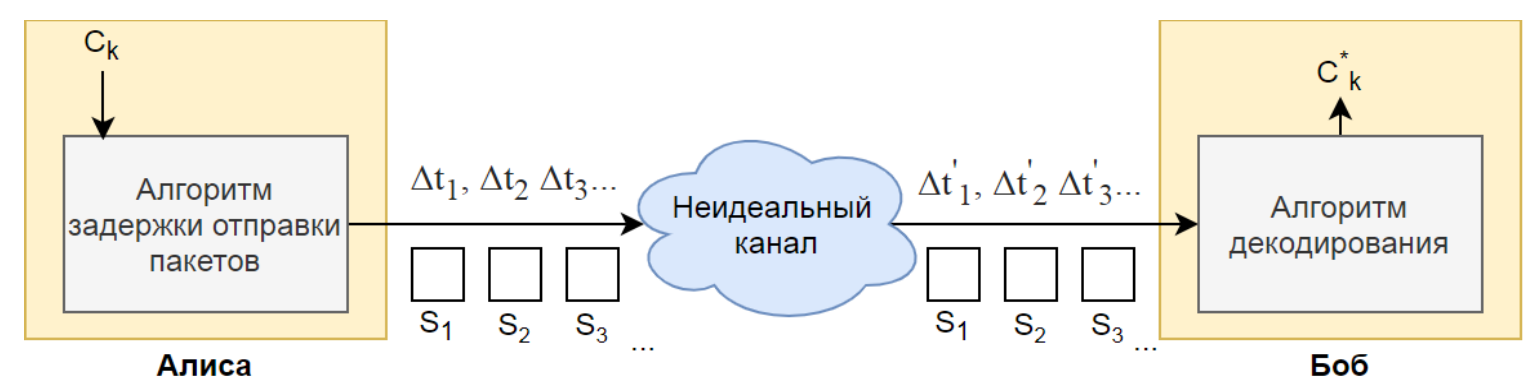

Рисунок 1: Схема скрытого канала, основанного на изменении межпакетных задержек.

В качестве наглядного примера, можно представить, что Алиса может выполнить инструкцию ping на компьютер Боба. Таким образом компьютер Боба получает последовательность істр пакетов с задержками между последовательными пакетами $\Delta \mathrm{t}^{\prime} 1$ секунд, $\Delta \mathrm{t}^{2} 2$ секунд, $\Delta \mathrm{t} 3$ секунд и так далее. [5]

С абстрактной точки зрения этот метод сводится к отправке символов источника через шумный канал и декодированию сообщения в месте назначения.

Фактически считается, что задержки на компьютере прибытия не совсем такие же, как на компьютере отправления из-за шума в Интернете. Любое устройство пересылки (маршрутизаторы, межсетевые экраны, коммутаторы) будет создавать небольшую задержку. Эта задержка будет изменяться в настоящем времени, что приводит к нарушению межпакетного времени и делает канал зашумленным.

В результате реализации метода на стороне Боба составляется таблица задержек, полученных при помощи анализатора сетевого трафика (например, Wireshark), которые в дальнейшем сравниваются со значениями в таблице кодировок, которую Бобу необходимо получить от Алисы.

Алисе, в ходе изучения сети, следует выбрать оптимальную систему кодировки, которая будет максимизировать пропускную емкость канала Шеннона, поскольку она устанавливает верхнюю границу максимального количества безошибочных цифровых данных.

Составляется определённая таблица задержек при отправлении пакетов получателю. Сравнивается вносимое значение задержки с результирующим при получении. Как только такая таблица будет заполнена, емкость Шеннона, представленная дискретным каналом без памяти, может быть оценена с помощью алгоритма Аримото-Блахута[6].

Поскольку характеристики сети постоянно меняются (например, появляются задержки или происходят перегрузки и т.д.), Алиса не сможет выбрать входное распределение, которое максимизирует пропускную способность канала для всех случаев. Таким образом, можно предположить, что ей лучше использовать некое универсальное входное распределение, которое хорошо работает для нескольких возможных каналов, при разных состояниях сети.

Способы обнаружения метода, основанного на преднамеренных временных задержках.

Первый метод [6], основанный на концепциях теории информации, требует глубокого изучения ситуации в сети. Необходимо провести ряд мероприятий по исследованию сети, с учетом того, что Алиса попытается выбрать распределение, которое максимизирует пропускную способность канала Шеннона.

Для этого необходимо воспользоваться следующими инструментами:

1. Сетевой монитор, который будет фиксировать фактическое распределение задержки между пакетами для каждого активного соединения. 
2. Алгоритм Аримото-Блахута, который, основываясь на результатах таблицы задержек, рассчитывает наилучшее распределение задержек ввода, учитывая пропускную способность канала Шеннона.

3. Алгоритм сходства, который сравнивает два распределения вероятностей на входных символах: одно полученное после мониторинга сетевого трафика и одно получено для максимизации пропускной способности канала при помощи алгоритма АримотоБлахута.

Второй метод [6] основан на простой идее, что если используется четное количество входных символов, число пакетов со средней задержкой очень мало по сравнению с максимальным количеством пакетов для любой заданной задержки. Статистический анализ межпакетных задержек предоставляет хорошие данные для классификации между обычным сетевым трафиком и трафиком, который обусловлен модуляцией межпакетных задержек.

Метод основан на предположении, что для канала с четным количеством входных символов межпакетные задержки будут сосредоточены вокруг двух различных значений (то есть двух различных задержек), в то время как в обычной связи, где задержки являются более или менее случайными, $\Delta \mathrm{t}$ распространяется вокруг одного значения (т.е. имеет нормальное распределение. (рисунок 2б).

Мы используем эту характеристику для статистического сравнения скрытого канала связи с обычным сетевым трафиком.
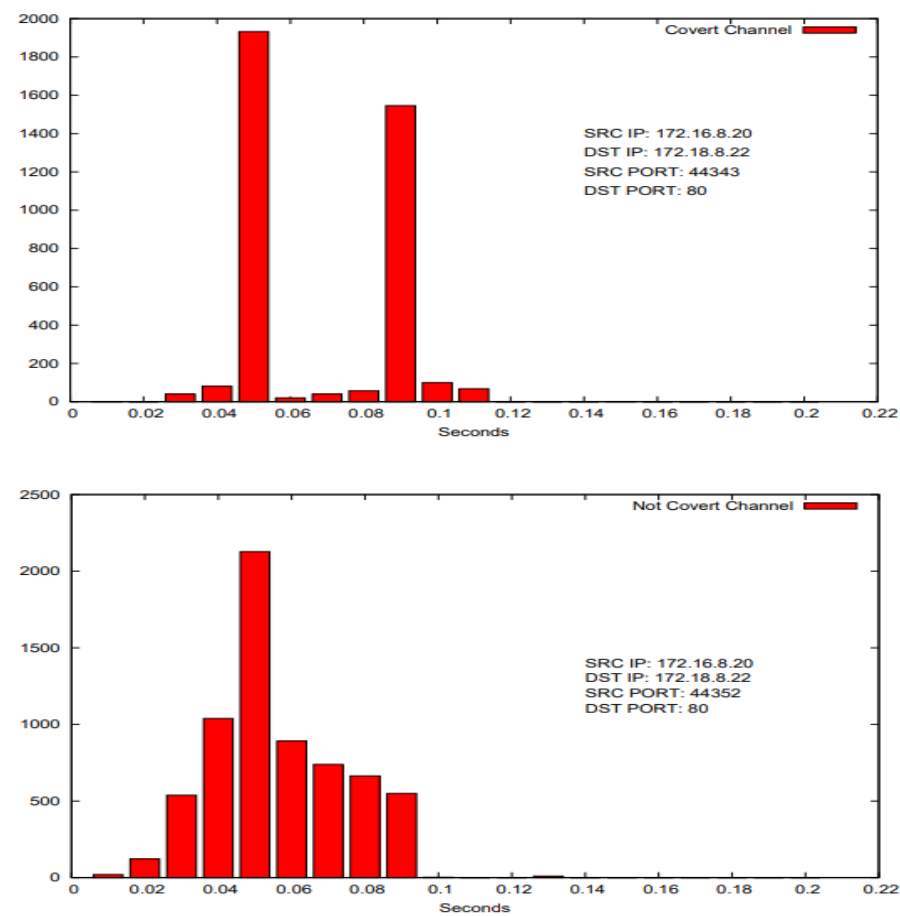

Рисунок 2 а) б): Гистограммы, характеризующчие статистический анализ задержек для скрытого и нормального канала, соответственно.

Глядя на рисунки 2а и 2б, разница между двоичным скрытым каналом и обычным трафиком становится очевидной. Для скрытого канала выборочное среднее значение $\mu$ межпакетных задержек будет где-то между двумя пиками. Следовательно, количество пакетов в гистограмме в этой точке будет очень низким. Однако, если взглянуть на нормальную схему трафика, среднее значение задержек между пакетами будет находиться в центре большого всплеска. В частности, чем меньше отношение $C_{\mu} /$ $C_{\max }$, тем выше вероятность наличия скрытого канала связи. Поэтому для расчета 
вероятности наличия скрытого канала мы можем воспользоваться следующей формулой:

$$
P_{\text {CovChan }}=1-\frac{C_{\mu}}{C_{\max }}
$$

где $C_{\mu}$ - это число пакетов, соответствующих средней задержке, а $C_{\max }$ - это число пакетов, соответствующее максимальной задержке.

При помощи IDE Intellij Idea и библиотеки Pcap4j можно реализовать экспериментальный макет, автоматизирующий подсчет вероятности наличия скрытого канала.

private Map<Float, Integer> getDelayPackets(PcapHandle handle, int size) throws NotOpenException \{ int countPackets $=0$; long

startTime

Map<Float, DecimalFormat while (true) \{

$$
\text { Packet }
$$

if (packet else \{

if

(countPackets

packet receivedPackets Integer> decimalFormat $=$ $=$

$=$ new $=$ $==$ startTime $=$ handle.getTimestamp ()$\cdot \operatorname{getTime}() ;\}$ else

long currentDelay $=($ startTime BigDecimal bigDecimal - handle. $=\quad$ new new new 0 HashMap $<>()$; DecimalFormat("\#.\#\#"); handle.getNextPacket(); receivedPackets.put(bigDecimal.setScale(2,RoundingMode.DOWN).floatValue(), receivedPackets.get(bigDecimal.setScale(2,RoundingMode.DOWN).floatValue ()$+1)$ ); $\}$ countPackets++;

if $($ countPackets $==$ size $)\{$ break; $\}\}$ return

Map<Float, Integer >

private float getCovertChannelChance(Map<Float, Integer> float resultDelaysSum $=0 ;$ int

for (Float delay : delayPackets.keySet()) \{ resultDelaysSum $+=$ for (Integer integer : delayPackets.values()) \{ if (integer $>\quad$ maxCount $\quad=$ integer; $\}$ return 1 - ((new BigDecimal(resultDelaysSum / size).setScale(2, RoundingMode.DOWN).floatValue()) / (maxCount)); \}

1. Белкина, Т. А. Аналитический обзор применения сетевой стеганографии для решения задач информационной безопасности / Т. А. Белкина. - Текст : непосредственный // Молодой ученый. 2018. № 11 (197). С. 36-44. [Электронный ресурс], URL: https://moluch.ru/archive/197/48821/ (дата обращения: 20.10.2020).

2. Wojciech Mazurczy, Milosz Smolarczyk, Krzysztof Szczypiorski Hiding Information in Retransmissions,in Telecommunication Systems 52(2), Маy 2009 [Электронный pecypc],URL: https://arxiv.org/ftp/arxiv/papers/0905/0905.0363.pdf (дата обращения 20.10.2020)

3. K. Ahsan, Covert Channel Analysis and Data Hiding in TCP/IP, M.A.Sc. thesis, Dept. of Electrical and Computer Engineering, University of Toronto, August 2002 , [Электронный pecypc],URL: https://github.com/emintham/Papers/blob/master/Ahsan,Kundur\%20Practical\%20Data\%20Hiding\%20in\%20TCP-IP.pdf (дата обращения:20.10.2020).

4. Костырин А.С., Красов А.В. “Обзор возможностей реализации канальной стеганографии на основе протоколов сетевого и транспортного уровней модели OSI. "//B сборнике: Актуальные проблемы инфотелекоммуникаций в науке и образовании (АПИНО 2017) Сборник научных статей VI Международной научно-технической и научно-методической конференции. Под редакцией С.В. 
Бачевского. 2017. C. 437-443. [Электронный pecypc] URL: https://www.elibrary.ru/item.asp?id=32578305 (дата обращения: 23.10.2020)

5. Красов А.В., Степанов Е.И. "Практическое применение сетевой стеганографии на примере протокола ICMP . " //Актуальные проблемы инфотелекоммуникаций в науке и образовании (АПИНО 2018) VII Международная научно-техническая и научно-методическая конференция. Сборник научных статей. В 4-х томах.Под редакцией С.В. Бачевского. 2018. С. 510-513. [Электронный pecypc], URL: https://www.elibrary.ru/item.asp?id=35231625 (дата обращения: 01.11.2020)

6. Vincent Berk, Annarita Giani, George Cybenko, Detection of Covert Channel Encoding in Network Packet Delays , Department of Computer Science - Dartmouth College Technical Report TR536, Revision 1 August 2005, revised November 2005 , [Электронный ресурc],URL:http://team.grayworld.net/cn/papers/detection_cc_encoding_network_pkts_delay_149.pdf(дата обращения: 20.11.2020).

\section{Митина О.А.}

Аналитические модели для решения задачи классификации данных

\section{МИРЭА - Российский технологический университет}

(Россия, Москва)

doi: $10.18411 / \mathrm{j}-02-2021-10$

idsp: ljournal-02-2021-10

\section{Аннотация}

Управление кредитным риском - основная задача банков и других кредитных организаций. Несвоевременные частичные или полные невозвраты тела кредита, а также процентной части в установленный соглашением срок и с соблюдением всех предусмотренных условий - одна из главных причин убытков финансовых учреждений.

Технологии интеллектуального анализа данных содержат эффективные средства построения скоринговых моделей - нейронные сети, деревья решений, а также логистическую регрессию для предсказания значения целевой переменной, позволяющей оценить кредитоспособность клиента.

Цель данной статьи - показать актуальность задачи классификации данных на примере финансово-кредитной сферы (кредитного займа).

Ключевые слова: нейронные сети; задачи классификации; логистическая регрессия, аналитическая платформа Loginom.

\section{Abstract}

Credit risk management is the main task of banks and other credit institutions. Untimely partial or complete non-repayment of the loan body, as well as the interest part, within the period established by the agreement and in compliance with all the conditions provided for, is one of the main causes of losses of financial institutions.

Data mining technologies contain effective tools for building scoring models - neural networks, decision trees, and logistic regression to predict the value of the target variable that allows you to assess the creditworthiness of the client.

The purpose of this article is to show the relevance of the problem of data classification on the example of the financial and credit sphere (credit loan).

Keywords: neural networks; classification problems; logistic regression, Loginom analytical platform.

Актуальность понятия «классификация» обусловлена ее всесторонним применением. Методы классификации задействуются при оценке кредитоспособности заемщиков (кредитном скоринге [1][2]), определении лояльности абонентов телекоммуникационных компаний, в торговле, медицинской диагностике и многих других сферах. 